\title{
Review of: "Nuclear RNA binding regulates TDP-43 nuclear localization and passive nuclear export"
}

\author{
Sen Yan
}

Potential competing interests: The author(s) declared that no potential competing interests exist.

This article studies the nuclear export of TDP-43 and the role of RNA in the localization of TDP-43 from multiple perspectives. It is proved that the nuclear export of TDP-43 is passive and is affected by the regulation of RNA in the body. But there are some problems that need to be improved. For example, this research is mainly carried out in vitro, so is it the same situation in vivo? In addition, most of the research is to explore the location of TDP-43 under normal circumstances rather than the disease state, and is there any limit to exploring the mechanism of diseases (such as ALS/FTD)? In general, the author has done a lot of work to provide new insights into the cytoplasmic shuttle mechanism of TDP-43.

1. Background line 99, “...allowing TDP-43 to diffuse from the nucleus in low-ATP conditions..." Why study the nuclear localization of TDP-43 under low ATP conditions?

2. Results line 119, “...Like actinomycin D (Suppl fig 1A-C), NVP-2 caused progressive, dose-dependent inhibition of RNA synthesis (Fig 1A-B),..." Actinomycin D and NVP-2 treatment concentrationdependently inhibited RNA synthesis, but why RNA puncta within nucleoli resulting from RNA polymerase I-dependent ribosomal RNA synthesis was retained. And why not use actinomycin D directly but use NVP-2.

3. Line 141, "TDP-43 exits the nucleus by passive diffusion". The author previously proved that inhibiting RNA Pol II promotes the nuclear export of TDP-43. Will temperature and ATP changes affect the activity of RNA Pol II?

4. Line 236, “In contrast to untreated controls, RNase treatment caused rapid TDP-43 nuclear efflux over 30 minutes even at $4{ }^{\circ} \mathrm{C}, \ldots .$. . In fig 4A, RNase treatment caused rapid TDP-43 nuclear efflux, but the cytoplasmic TDP-43 is not seen in the figure.

5. Line 550, “The observation that GU-rich nuclear pre-mRNAs critically regulate TDP-43 nuclear localization raises the possibility that disruption of nuclear RNA metabolism could contribute to TDP-43 nuclear clearance in ALS/FTD...". The author raises the possibility that disruption of nuclear RNA metabolism could contribute to TDP-43 nuclear clearance in ALS/FTD. This study mainly studies the nuclear localization of TDP-43 under normal conditions. We know that some ALS/FTD patients have TDP -43 mutation, then what is the nuclear localization of TDP-43 in the TDP-43 mutation and disease state? 
